\title{
EQUINODERMOS COLECTADOS POR EL OCEANOGRAFICO PESQUERO \\ "B.C. ACADEMIC KNIPOVIC", EN EL SUR DE CHILE
}

ALFONSO GUZMÁN C.

Universidade de Chile, Osorno, Chile

\section{SYNOPSIS}

This is a study of the echinoderms caught during the oceanographic fishery cruise performed by the "B.C. Academic Knipovic" in April, 1973. The recorded specimens are from the South of Chile $\left(45^{\circ} 08\right.$ 'S; $\left.74^{\circ} 54^{\prime} \mathrm{W}\right)$ and belong to the classes Asteroidea: Ctenodiscus procurator Sladen; Peribolaster folliculatus Sladen; Porania antarctica Smith; Echinoidea: Tripylaster philippi (Gray) and Ophiuroidea: Astrotoma agassizzi Lyman and Gorgonocephalus chilensis (Philippi). New recorded to Peribolaster folliculatus Sladen, $45^{\circ} 08^{\prime} S$; $74^{\circ} 54^{\prime} W$. Ecological data, geo. graphical and batimetric distribution are given.

\section{Introduccion}

El Instituto Fomento Pesquero (IFOP), realiza en abril, 1973 una pesca exploratoria por el Sur de Chile, en el N/Oc. pesquero "B.C. Academic Knipovic"; oportunidad en que se obtienen equinodernos en la estación bentica $\left(45^{\circ} 08^{\prime} \mathrm{S} ; 74^{\circ} 54 \mathrm{~W}\right)$ pertenecientes a las clases: Asteroidea, Echinoidea y Ophiuroidea; material que sirve para actualizar la fauna y analizar su distribución geográfica.

\section{Resultados}

Los ejemplares de equinodermos fueron obtenidos a $161 \mathrm{~m}$ de profundidad en sustrato fangoso.

Se indica la familia, especie, una sinonimia, radio mayor(R) y menor ( $\mathrm{r}$ ) de un sólo especimen, diagnosis, distribución geográficabatimetrica y la cantidad de ejemplares analizados por especie.

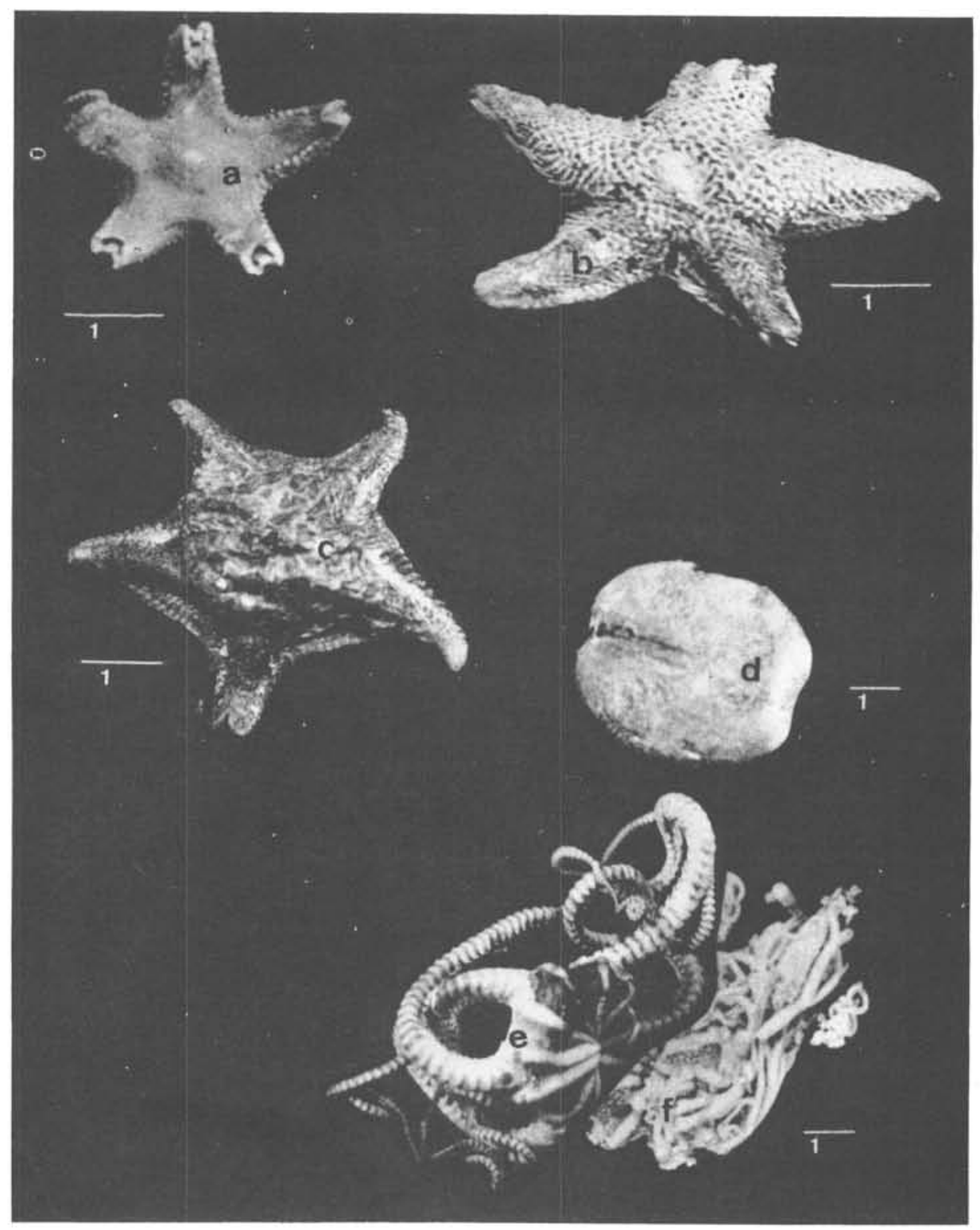

Fig. 1. a: Ctenodiscus procurator Sladen; b: Peribolaster folliculatus Sladen; c: Porania antarctica Smith; d: Tripylaster philippi (Gray); $\mathrm{e}:$ Astrotoma agassizzi Lyman; $\mathrm{f}$ : Gorgonocephalus chilensis (Philippi). 


\section{ASTEROIDEA}

Fam. GONIOPECTINIDAE

Ctenodiscus procurator Sladen, 1889

(Fig. 1a)

1889 Ctenodiscus procurator Sladen, p.173

Diagnosis. $\mathrm{R}=31 \mathrm{~mm}$ y $\mathrm{r}=16 \mathrm{~mm}$

Area abactinal paxiliforme, placas superomarginales gruesas, cada placa lleva en su borde superior una púa cónica; presenta fasciolas entre las placas marginales.

Distribucion Geografica. Archipielago chileno hasta Cabo de Hornos; Atlantico ( $\left.45^{\circ} 02^{\prime} \mathrm{S} ; 61^{\circ} 18^{\circ} \mathrm{W}\right)$ Cabo Dos Bahías.

Distribucion Batimetrica: 0 - $2385 \mathrm{~m}$

Material Examinado: 2

Fam. KORETHRASTERINAE

Peribolaster folliculatus Sladen, 1889

(Fig. 1b)

1889 Peribolaster folliculatus Sladen, p. 464

Diagnosis. $\mathrm{R}=39 \mathrm{~mm} \mathrm{y} \mathrm{r}=19 \mathrm{~mm}$

Esqueleto abactinal con placas cruciformes, reticulado de mallas grandes. Cada placa posee un grupo de púas de tamaño similar. Placas orales grandes.

Distribucion Geografica: Peninsula Tres Montes (Chile) a Malvinas (Argentina), Sur de Africa; Sladen menciona como especie rara, indicando sólo la localidad tipo $\left(45^{\circ} 54^{\prime} \mathrm{S} ; 75^{\circ} 12^{\prime} \mathrm{W}\right)$, como único registro; amplía su distribución septentrionalmente a $45^{\circ} 08^{\prime} \mathrm{S} ; 74^{\circ} 54^{\prime} \mathrm{W}$.

Distribucion Batimetrica: $81-161 \mathrm{~m}$

Material Examinado: 4

\section{Fam. PORANIIDAE}

Porania antarctica Smith, 1876

(Fig. 1c)

1931 Porania antarctica Fisher, p.3

Diagnosis: $\mathrm{R}=41 \mathrm{~mm} \mathrm{y} \mathrm{r}=21 \mathrm{~mm}$

Forma pentagonal, area abactinal con piel gruesa que oculta las placas. Placas infero-marginales bien nítidas (2-3 púas).

Distribucion geografica: Desde Provincia de Buenos Aires; Antartica hasta $30^{\circ} \mathrm{S}$ (Chile).

Distribucion Batimetrica: $0-320 \mathrm{~m}$

Material Examinado: 4

\section{ECHINOIDEA}

Fam. HEMIASTERIDAE

Tripylaster philippi (Gray, 1851)

(Fig. 1d)

1953 Tripylaster philippi Bernasconi, p. 53

Diagnosis: Caparazón ovalado, algo aplanado con escotadura pronunciada en la parte anterior. Cara aboral convexa, truncada en la parte posterior. Púas grandes espatuladas, pedicelarios globíferos $\mathbf{y}$ tridentados.

Distribucion Geografica: Rio La Plata (Argentina) hasta Puerto Montt (Chile).

Distribucion Batimetrica. 0 - $162 \mathrm{~m}$

Material Examinado: 3

\section{OPHIUROIDEA}

Fam. GORGONOCEPHALIDAE

Astrotoma agassizzi Lyman, 1875

(Fig. 1e)

1875 Astrotoma agassizzi Lyman, p. 24

Diagnosis: Disco grande, diez barras bien nítidas divergentes en el extremo distal. Brazos simples alargados, ganchos microscópicos en la unión de los segmentos braquiales.

Distribucion Geografica: De Puerto Montt al Estrecho de Magallanes; Islas Georgias del Sur; Indopacífico; Islas Subantarticas y la Antártica.

Distribucion Batimetrica. 3-1000 m

Material Examinado: 6

Gorgonocephalus chilensis (Philippi, 1858)

(Fig. 1f)

1880 Gorgonocephalus chilensis Lyman, p. 43

Diagnosis: Escudos radiales con barras angostas, brazos bastantes ramificados, presentando la primera bifurcación dentro del margen del disco.

Distribucion Geografica: Sur de Argentina ( $\left.36^{\circ} \mathrm{S}\right)$; Malvinas, Georgias del Sur; Pacífico: $33^{\circ} \mathrm{S}$ hasta el Sur de Chile.

Distribucion Batimetrica: 8 - $411 \mathrm{~m}$

Material Examinado: 3

\section{Conclusiones}

Se entregan los nuevos antecedentes sobre los equinodermos estudiados:

1. Las especies corresponden Asteroideos: Ctenodiscus procurator Sladen; Peribolaster folliculatus Sladen; Poranta antarctca Smith; Equinoidea: Tripylaster philippi (Gray); y Ofiuroideos: Astrotoma agassizzi Lyman y Gorgonocephalus chilensis (Philippi).

2. La presencia de esta fauna nos indica que pertenecen al tipo de aguas frías.

3. Sladen (1889), señala Peribolaster folliculatus como especie rara, indicando sólo la localidad tipo ( $\left.45^{\circ} 53^{\prime} \mathrm{S} ; 75^{\circ} 12^{\prime} \mathrm{W}\right)$; amplía su distribución septentrionalmente a $45^{\circ} 08^{\mathrm{S}} \mathrm{S} ; 74^{\circ} 54 \mathrm{~W}$.

\section{Agradecimientos}

Expreso mis sinceros agradecimientos a la Dra. I. Bernasconi, del Museo Argentino de Ciencias Naturales "Bernardino Rivadavia", por sus valiosas informaciones y sugerencias.

\section{Referencias bibliográficas}

BERNASCONI, I. 1953. Monografía de los equinoídeos argentinos. An. Mus. nac. Hist. nat. B. Aires, 2 ? ser., $6(2): 53-56$, lam. XXVII.

FISHER, W. 1931. Report on the South American sea stars collected by Waldo Schmitt. Proc. U.S. natn. Mus., $78: 3$.

GRAY, J.E. 1851. Catalogue of the recent Echinida in the collection of the British Museum. I - Echinida Irregularia. London. p. 59 , pl. V.

LYMAN, Th. 1875. Ophiuridae and Astrophytidae. Zoological results of the Hassler Expedition, Part II, III. Cat. Mus. comp. Zool., Harv., 8(3) : 1-34, 5 pls.

- 1880. Preliminary list of living Ophiuridae and Astrophytidae. Bull. Mus. comp. Zool., Harv., 10(6) : 229.

PHILIPPI, R. 1858. Beschreibung einiger neuen Seesterne aus dem Meere von Chiloe. Arch. Naturgesch., $24: 1$.

SLADEN, P. 1889. Report on the asteroid collected by H.M.S. Challenger during the years 1873-76. Rep. scient. Results Voyage H.M.S. Challenger, Zool., $30: 173-464$.

SMITH, E. 1876. Description of species of Asteriidae in the British Museum. Ann. Mag. nat. Hist., (4) : 17. 